\title{
Intervenção em habilidades cognitivas e metacognitivas de leitura em alunos do Programa de Educação Tutorial - PET
}

\author{
Marisa Cosenza Rodrigues' \\ Marília Aparecida Pontes Alves \\ Rafaela Duque de Almeida \\ Renata de Lourdes Miguel da Silva
}

Universidade Federal de Juiz de Fora, Juiz de Fora - MG - Brasil

\begin{abstract}
Resumo: A compreensão leitora é essencial para uma formação acadêmica de qualidade. $O$ estudo objetivou avaliar o efeito de um programa dirigido a promover o desenvolvimento de estratégias cognitivas e metacognitivas de leitura em II alunos do curso de Psicologia, bolsistas do Programa de Educação Tutorial, os quais foram pré e pós-avaliados por meio da escala de estratégias metacognitivas de leitura - formato universitário. O programa envolveu 12 encontros semanais, num total de 24 horas, tendo como base a conjugação de três pilares de estratégias metacognitivas - AIM, K-W-L e K-W-L PLUS. Encontraram-se médias superiores na pós-avaliação, com resultados significativos para os três fatores que compõem a escala (global, suporte e solução de problemas), assim como para o escore geral. Os dados sugerem aprimoramento da utilização de estratégias cognitivas e metacognitivas de leitura após a intervenção, realçando a importância de estudos dessa natureza com universitários.
\end{abstract}

Palavras-chave: compreensão da leitura; estudantes universitários; estudos de intervenção; metacognição; promoção.

\section{INTERVENTION IN COGNITIVE AND METACOGNITIVE READING SKILLS IN EDUCATION PROGRAM TUTORIAL - PET STUDENTS}

\begin{abstract}
Reading comprehension is essential for quality academic education. The aim of this study was to evaluate the effect of a program aimed at promoting the development of cognitive and metacognitive reading strategies in II students of the Psychology course, with scholarships on the Tutorial Education Program. These students were pre- and post-assessed by the metacognitive reading strategies scale (university format). The program involved I 2 weekly meetings, totaling 24 hours based on a combination of three pillars of metacognitive strategies - AIM, K-W-L and K-W-L PLUS. Higher averages were found in the post-evaluation, with significant results for the three factors that make up the scale (global, support and problem solving), as well as for the overall score. The data suggests better use of cognitive and metacognitive reading strategies after the intervention, highlighting the importance of such studies with university students.
\end{abstract}

Keywords: reading comprehension; university students; intervention studies; metacognition; fostering.

1 Endereço para correspondência: Marisa Cosenza Rodrigues, Praça Jarbas de Lery Santos, 37, ap. 103, São Mateus - Juiz de Fora - MG - Brasil. CEP: 36.016-390. E-mail: rodriguesma@terra.com.br. 
INTERVENCIÓN EN HABILIDADES COGNITIVAS Y METACOGNITIVAS DE LECTURA EN LOS ESTUDIANTES DEL PROGRAMA DE EDUCACIÓN TUTORIAL - PET

\begin{abstract}
Resumen: La comprensión lectora es esencial para una formación académica de calidad. El objetivo del estudio fue evaluar el efecto de un programa direccionado a promoción del desarrollo de estrategias cognitivas e meta-cognitivas de lectura en II estudiantes del curso de Psicología, becados del programa de Educación Tutorial, ellos que fueran pre y pos-evaluados por la escala de estrategias meta-cognitivas de lectura - formato universitario. El programa incluyó 12 sesiones por semanas, por un total de 24 horas basado en la conjugación de 3 columnas de estrategias meta-cognitivas - AIM, K-W-L e K-W-L PLUS. Fueron encontradas medias superiores en la post-evaluación, con resultados significativos para los tres factores que componen la escala (general, el apoyo y solución de problemas) así como para el escore general. Los datos sugieren mejora de la utilización de estrategias cognitivas e meta-cognitivas de lectura después a intervención, destacando la importancia del estudio de tal naturaleza con universitarios.
\end{abstract}

Palabras clave: comprensión de lectura; estudiantes universitarios; estudios de intervención; metacognición; promoción.

A leitura está presente em diversos contextos da vida do ser humano. Tanto no contexto acadêmico como no âmbito profissional ou pessoal, a leitura fomenta a aprendizagem e o aprimoramento das relações sociais, constituindo um processo complexo e ativo no qual estão envolvidos vários aspectos do desenvolvimento psicológico, como capacidade sensorial, percepção, motivação, pensamento, memória, entre outros (Cantalice \& Oliveira, 2009).

No que tange ao ensino superior, sua relação apresenta-se como singular, pois trata-se de atividade central em várias disciplinas e seu domínio é essencial para uma formação acadêmica de qualidade. Nesse aspecto, converge com as diretrizes do Programa de Educação Tutorial (PET) do Ministério da Educação (MEC) que busca propiciar e viabilizar aos alunos de graduação, por meio de atividades extracurriculares orientadas por um tutor, uma formação acadêmica complementar conjugando, de forma articulada, ensino, pesquisa e extensão. De acordo com o Ministério da Educação (2006), as atividades extracurriculares inseridas no programa buscam contemplar experiências não presentes nas estruturas curriculares tradicionais, visando a uma formação global que favoreça a trajetória acadêmica, tanto para a inserção produtiva no mercado profissional quanto para o desenvolvimento de estudos de pós-graduação.

Mesmo constituindo uma habilidade crucial, vários estudos têm demonstrado (Calderón-Ibáñez \& Quijano-Peñuela, 2010; Santos, Suehiro, \& Oliveira, 2004; Silva \& Witter, 2008) que a compreensão leitora dos universitários está aquém do que seria desejável para a formação acadêmica no ensino superior, encontrando um número expressivo de estudantes que não conseguem abstrair de forma satisfatória a informação lida. Cantalice e Oliveira (2009) alertam para essa realidade preocupante por se tratar de futuros profissionais no mercado de trabalho e que poderão sair da universidade, se nada for feito, com uma qualificação técnica insuficiente para os desafios inerentes à profissão.

Segundo Oliveira e Santos (2005), a realização de uma leitura não é uma tarefa simples. Não basta decodificar, é necessário que o leitor se contextualize e atribua 
significado à sua leitura. É essencial uma interação leitor/texto/autor para que a compreensão e a aprendizagem ocorram efetivamente. Nesse contexto, as estratégias de leitura ocupam uma posição relevante.

As estratégias de leitura podem ser cognitivas ou metacognitivas e se caracterizam por serem planos flexíveis usados pelos leitores para aprimorar a capacidade de compreensão. As estratégias de leitura cognitivas são definidas como comportamentos e pensamentos que influenciam na aquisição da informação lida. As metacognitivas envolvem, por sua vez, a consciência que o leitor tem sobre o próprio nível de compreensão e o controle de ações cognitivas por meio de estratégias que facilitem a compreensão de um determinado tipo de texto. Por meio da metacognição, o leitor é capaz de planejar, monitorar e regular o seu próprio pensamento antes, durante e após a leitura (Cantalice \& Oliveira, 2009; Kopcke, 1997).

Para Kopcke (1997), o ingresso na vida universitária não garante que os estudantes possam desenvolver por si mesmos habilidades de compreensão de textos, sendo necessário intervir a fim de ensinar-lhes a observar em que situações a compreensão ficou prejudicada e o que pode ser feito para reparar e retomar o processo de entendimento. O referido autor, com base nas propostas pioneiras de outros estudiosos, indica para a intervenção a conjugação de três pilares de estratégias metacognitivas conhecidas originalmente por AIM, K-W-L e K-W-L PLUS. A estratégia author's intended message (AIM), ou seja, mensagem pretendida pelo autor, tem por objetivo geral habilitar os universitários a ler um texto expositivo de maneira eficaz e autônoma, por meio de perguntas específicas que são formuladas antes, durante e após a leitura. A estratégia K-W-L visa desenvolver a leitura de textos teóricos partindo do conhecimento que o leitor já possui sobre o assunto. À semelhança da AIM, anteriormente descrita, a K-W-L envolve atividades anteriores, simultâneas e posteriores à leitura e abarca três questões:

- Fase K (What I know?): O que sei sobre o assunto?

- Fase W (What I want to know?): O que pretendo saber?

- Fase L (What I learned?): O que aprendi com a leitura?

Por sua vez, a estratégia K-W-L PLUS acrescenta à fase K-W-L, anteriormente citada, dois complementos: um mapa do texto e um resumo. O mapa do texto, nos moldes de um diagrama, centraliza a ideia-chave e aglutina outros conceitos nucleares contidos nele, funcionando como um guia para a elaboração posterior do resumo.

De acordo com Marini (2006), leitores pouco hábeis raramente utilizam estratégias metacognitivas de leitura para auxiliar a compreensão, enquanto os leitores habilidosos lançam mão mais frequentemente de uma variedade de estratégias conforme a complexidade do texto a ser lido. O estudo de Song (1998), por exemplo, revelou que leitores hábeis são melhores no monitoramento da sua compreensão quando comparados aos menos hábeis, sendo mais conscientes das estratégias que empregam e usando-as mais flexivelmente e com eficácia. 
Estudos nacionais que têm se dedicado a explorar a utilização de estratégias metacognitivas de leitura pelos alunos (Cantalice \& Oliveira, 2009; Joly, 2007; Joly, Santos, \& Marini, 2006) demonstram que, de uma forma geral, os estudantes recorrem às estratégias de leitura. Contudo, a frequência com que são utilizadas tais estratégias está abaixo do que se espera para um bom leitor, e o momento de uso ocorre especialmente durante a leitura, revelando que grande parte desses leitores as utiliza apenas quando se deparam com dificuldade de compreensão leitora.

A despeito de sua relevância, como salientam Oliveira e Santos (2008), há poucas pesquisas envolvendo programas de intervenção para o desenvolvimento da leitura quando comparadas aos estudos de levantamento ou correlacionais, embora aquelas demonstrem resultados animadores tanto no âmbito internacional quanto nacional. Como afirmam Joly e Marini (2006), há um interesse crescente de pesquisadores internacionais pelo ensino de estratégias de leitura, bem como dos seus efeitos específicos sobre a compreensão leitora. Nessa direção, Espinosa e Tamayo (2009) revisaram pesquisas com intervenção publicadas entre 1996 e 2008 sobre o ensino de estratégias metacognitivas de leitura e seu impacto sobre a compreensão de leitura. As informações distribuíram-se em três categorias de análise: os programas de intervenção, os métodos instrucionais e outros aspectos metodológicos (tipo de experimento, os instrumentos, as amostras). A investigação indicou uma melhora significativa da compreensão leitora dos estudantes após participação nos programas. Os autores concluíram ainda que a evolução da compreensão se deve sobretudo à tomada de consciência metacognitiva durante a leitura, independentemente da estratégia metacognitiva privilegiada nas intervenções, ou seja, o aluno participante de um programa de intervenção em leitura torna-se mais consciente quanto à importância de ser ativo no processo de leitura para que atinja uma efetiva compreensão textual.

Falk-Ross (2002), por exemplo, avaliou o efeito de um programa de ensino de estratégias de leitura sobre o desempenho de quatro alunos universitários considerados maus leitores, com idade entre 18 e 22 anos. A comparação de desempenho na avaliação pré e pós-programa indicou diferenças estatisticamente significativas no que tange à compreensão leitora desses alunos. Constatou-se que o uso de estratégias específicas implementadas antes (exploração dos objetivos da leitura e de aspectos da organização textual), durante (trabalho de revisão e clarificação das informações existentes) e após a leitura (elaboração do resumo e transposição das informações para as atividades) possibilitou aos alunos uma integração entre as habilidades previamente aprendidas na direção de uma leitura significativa e eficaz com as demandas oriundas dos cursos universitários. Verificou-se também que esses estudantes tornaram-se mais focados, produtivos e críticos em suas tarefas após o programa de intervenção, colaborando de forma significativa nas discussões em sala de aula.

Diante da carência de instrumentos validados voltados especificamente para a avaliação das estratégias de leitura em nosso meio, a quase totalidade dos estudos com intervenção encontrados na literatura também focaliza a investigação dos possíveis 
efeitos indiretos das intervenções sobre a compreensão leitora e mediante a utilização da técnica do Cloze (Coelho \& Correa, 2010; Oliveira \& Santos, 2008; Sampaio \& Santos, 2002). Como informam Joly e Marini (2006), esse método, criado por Taylor em 1953, vem sendo utilizado de maneira recorrente para avaliar a compreensão em leitura de alunos dos ensinos básico e superior. Contudo, tendo em vista a necessidade de avaliar a utilização de tais estratégias em nossa realidade, Joly (2004) tem se dedicado a elaborar e validar um instrumento para universitários - a escala de estratégias metacognitivas de leitura - formato universitário (EMeL-U) -, obtendo importantes evidências de validade (Joly, 2007; Joly, Cantalice, \& Vendramini, 2004). Salienta-se que, até o momento, com exceção do presente estudo, não foram encontradas pesquisas com intervenção nacionais que utilizaram o referido instrumento para avaliar, de modo mais específico, as estratégias metacognitivas de leitura.

No que tange aos efeitos sobre a compreensão leitora, Oliveira e Santos (2008) conduziram um estudo de intervenção com a participação de 35 universitários brasileiros com média de 21,4 anos de idade, iniciantes de um curso de Administração de Empresas que frequentaram a disciplina Leitura e Produção de Textos (LPT), os quais foram pré e pós-avaliados quanto à compreensão em leitura por meio da técnica do Cloze. A intervenção envolveu 17 semanas (68 horas), com utilização de materiais didáticos e gêneros textuais diversificados. Os resultados evidenciaram um efeito positivo do programa com diferença estatisticamente significativa quanto à habilidade de compreensão textual dos participantes quando comparadas as etapas pré e pós-intervenção. Ainda que tal mudança de desempenho tenha se mostrado discreta, os dados sugerem que os estudantes tendem a se beneficiar de programa de remediação/prevenção voltado para o aprimoramento da compreensão leitora.

Sampaio e Santos (2002), por sua vez, implementaram e avaliaram um programa de intervenção em leitura e redação de textos, com 42 alunos ingressantes de dois cursos universitários. Não foram encontradas diferenças significativas entre pré e pós-avaliação no que se refere à compreensão leitora, contudo constataram-se mudanças qualitativas por meio de comentários dos docentes que lecionavam para a mesma turma e quanto às atitudes positivas dos alunos em relação à importância da leitura e da redação em sua vida acadêmica e profissional.

No âmbito do ensino médio, Coelho e Correa (2010) examinaram o progresso da compreensão leitora de adolescentes por meio do desenvolvimento da habilidade de monitoramento da leitura. Participaram 62 alunos do primeiro ano do ensino médio, distribuídos nos grupos experimental e de controle. Nesse estudo, os autores utilizaram o teste de Cloze para pré e pós-avaliar a compreensão leitora. A metodologia do paradigma de detecção de erros foi utilizada para as tarefas de monitoramento na pré-avaliação, na intervenção e na pós-avaliação. Após a intervenção, o grupo experimental mostrou-se significativamente melhor do que o controle nas tarefas de leitura (Cloze) e monitoramento, sugerindo que as habilidades de compreensão leitora e de monitoramento se influenciam, além de serem passíveis de treinamento. 
Esses indicadores positivos permitem considerar o quanto é desejável que a universidade proporcione aos alunos programas que visem remediar ou prevenir suas dificuldades em leitura por meio do desenvolvimento de habilidades específicas. Como lembra Witter (1997), a universidade, além de ser responsável pela formação de futuros profissionais, é a última oportunidade formal para o desenvolvimento de leitores críticos e competentes na apropriação das informações lidas.

Com o propósito de conjugar os interesses do PET e dos programas de intervenção em leitura no ensino superior, o presente estudo teve como objetivo implementar e avaliar (projeto piloto) o efeito de uma intervenção dirigida a promover a utilização de estratégias de leitura de alunos bolsistas do PET do curso de Psicologia da Universidade Federal de Juiz de Fora (UFJF/MG).

\section{Método}

\section{Participantes}

Participaram da pesquisa 11 alunos bolsistas (2011) do PET-Psicologia da UFJF, dez do sexo feminino e um do sexo masculino, com média de 22,6 anos de idade.

\section{Instrumento e materiais}

Utilizou-se a EMeL-U (Joly, 2004), composta por 35 itens do tipo Likert de resposta (nunca, 1 ponto; poucas vezes, 2 pontos; algumas vezes, 3 pontos; muitas vezes, 4 pontos; sempre, 5 pontos). A escala tem por objetivo avaliar o tipo e a frequência de estratégias de leitura que o aluno universitário utiliza nos intervalos antes, durante e após a leitura de textos acadêmicos. As estratégias metacognitivas de leitura estão divididas em três fatores. As estratégias metacognitivas de leitura globais $(1,2,3,4,5$, $6,7,9,16$ e 26) representam um conjunto de estratégias de leitura orientadas para uma análise geral do texto. As estratégias metacognitivas de leitura de suporte (11, $12,14,15,18,21,24,30,32$ e 35) dão apoio ao leitor para compreender a informação por meio do uso de materiais de referências, grifos, entre outros. E as estratégias metacognitivas de leitura de solução de problemas $(8,10,13,17,19,20,22,23,25,27,28$, 29, 31, 33 e 34) são utilizadas quando surgem dificuldades de compreensão para o leitor ante as informações presentes no texto.

Para a implementação do programa, utilizaram-se materiais didáticos envolvendo as modalidades de resenha, resumo e fichamento, capítulos de livros e artigos científicos na área da psicologia, poemas, poesias e matérias jornalísticas de cunho educacional. Utilizaram-se ainda folhas com perguntas a serem respondidas antes, durante e após a leitura dos textos trabalhados a cada encontro, bem como uma folha de registro dividida em quatro colunas, com as seguintes perguntas: "O que sei sobre o assunto?", "Que categorias de informação espero utilizar?", "O que pretendo encontrar" e "O que aprendi e ainda preciso aprender sobre o assunto?", com base no que é proposto por Kopcke (1997). 


\section{Procedimentos}

O projeto foi aprovado pelo Comitê de Ética em Pesquisa da UFJF (Registro CEP-UFJF n²356.096.2011). Realizou-se o convite aos alunos petianos de psicologia para a participação na pesquisa. Após serem informados dos objetivos da pesquisa, os alunos assinaram o Termo de Consentimento Livre e Esclarecido. Em seguida, realizou-se a pré-avaliação dos alunos mediante a aplicação da EMeL-U (Joly, 2004), e definiram-se dia, horário e local para implementação do programa de leitura no Centro de Psicologia Aplicada (CPA) da UFJF.

O programa foi realizado mediante 12 encontros, totalizando 24 horas com um encontro semanal. Os textos foram pré-selecionados e explorados a partir de uma conjugação de atividades envolvendo os três pilares de estratégias metacognitivas: AIM, K-W-L e K-W-L PLUS (Kopcke, 1997), já descritas anteriormente. O programa subdividiu-se em três etapas: 1. utilização da estratégia AIM, em que se trabalhou, de forma complementar, a elaboração de paráfrases (seis encontros), 2. utilização da estratégia K-W-L (três encontros) e 3) exploração da estratégia K-W-L PLUS (três encontros).

Ao término da intervenção, realizou-se a pós-avaliação dos participantes, seguindo o mesmo padrão da pré-avaliação. Os dados obtidos foram tabulados e analisados.

\section{Resultados}

Na descrição das variáveis métricas, utilizaram-se médias e desvio padrão. A fim de comparar a diferença nos escores dos participantes nas etapas de pré e pós-avaliação, foi empregado o teste $t$ de Student para amostras pareadas. Nesse caso, aplicaram-se testes paramétricos por obter, pela prova de Kolmogorov-Smirnov, normalidade na maior parte das variáveis. O nível de significância considerado foi $p \leq 0,05$.

Com o objetivo de verificar se houve diferença significativa nas etapas de pré e pós-avaliação da intervenção, compararam-se os escores obtidos nos três fatores da escala utilizada (fator 1: leitura global; fator 2: leitura de suporte; fator 3: leitura de solução de problemas) e no escore geral dos participantes nos dois momentos. A Tabela 1 apresenta as médias e os desvios padrão obtidos nas etapas de pré e pós-avaliação.

\section{Tabela I. Médias e desvios padrão obtidos nas etapas de pré e pós-avaliação}

\begin{tabular}{lcccc} 
& \multicolumn{2}{c}{ Pré-avaliação } & \multicolumn{2}{c}{ Pós-avaliação } \\
\cline { 2 - 5 } Escala & $M$ & DP & M & DP \\
\hline Fator global & 32,64 & 8,32 & 38,55 & 3,29 \\
Fator suporte & 33,27 & 7,57 & 40,09 & 5,22 \\
Fator solução de problemas & 48,27 & 7,51 & 58,55 & 8,66 \\
Escore geral & 114,18 & 22,32 & 137,18 & 16,53 \\
\hline
\end{tabular}

Fonte: Elaborada pelas autoras. 


\section{Discussão}

No que se refere ao escore geral, verifica-se que houve uma tendência de aumento nas médias da pós $(M=137,18)$ em relação à pré-avaliação $(M=114,18)$.

$\mathrm{O}$ teste $\mathrm{t}$ de Student para amostras pareadas evidenciou diferença significativa no nível de 0,05 para os três fatores da escala, a saber: leitura global $(t=2,67 ; p=0,002)$, leitura de suporte $(t=4,17 ; p=0,002)$ e leitura de solução de problemas $(t=4,31 ; p=$ $0,002)$, bem como para o escore geral $(t=4,39 ; p=0,001)$. Mais especificamente, os participantes apresentaram médias superiores quanto à utilização de estratégias metacognitivas de leitura na etapa de pós-avaliação, indicando que o programa implementado apresentou um efeito positivo no que tange ao desenvolvimento de estratégias cognitivas e metacognitivas de leitura. Esse dado converge na direção de outros estudos de intervenção (Coelho \& Correa, 2010; Falk-Ross, 2002; Oliveira \& Santos, 2008), os quais também indicaram benefícios para os participantes após a intervenção focalizada no desenvolvimento de tais estratégias.

Quanto aos três fatores que compõem a escala (global, suporte e solução de problemas), também é possível observar um aumento nas médias. Os dados apontam ainda que os estudantes recorrem com maior frequência às estratégias metacognitivas de solução de problemas, tanto na pré $(M=48,27)$ como na pós-avaliação $(M=58,55)$, corroborando estudos anteriores (Cantalice \& Oliveira, 2009; Joly, 2007; Joly et al., 2006). Cabe ressaltar que os acadêmicos teriam uma leitura mais eficiente ao utilizarem as estratégias metacognitivas global e de suporte tanto quanto utilizam as estratégias de solução de problemas, revelando-se um leitor habilidoso, que usa com frequência uma variedade de estratégias conforme a complexidade do texto a ser lido (Marini, 2006).

Diante dos resultados obtidos no presente estudo, verifica-se a importância de programas de intervenção para potencializar a compreensão leitora dos estudantes por meio do ensino de estratégias cognitivas e metacognitivas de leitura.

A pesquisa, por um lado, atende a uma demanda apontada em vários estudos que é a necessidade de programas de intervenção em leitura na universidade e, por outro, converge com as diretrizes do PET, que visa garantir uma melhor qualificação dos acadêmicos por meio de atividades extracurriculares.

O ensino de estratégias cognitivas e metacognitivas de leitura necessita ser realizado desde os primeiros anos de formação escolar para que o aluno aprenda desde cedo a fazer uso da metacognição, tendo em vista uma compreensão leitora mais competente e eficaz. A contribuição da universidade é fundamental no sentido de capacitar professores dos ensinos fundamental e médio, bem como minimizar as dificuldades encontradas pelos alunos por meio da implementação de programas que viabilizem o desenvolvimento da habilidade leitora. 


\section{Referências}

Calderón-Ibáñez, A., \& Quijano-Peñuela, J. (2010). Características de comprensión lectora en estudiantes universitarios. Estudios Socio-Jurídicos, 12(1), 337-364.

Cantalice, L. M., \& Oliveira, K. L. (2009). Estratégias de leitura e compreensão textual em universitários. Psicologia Escolar e Educacional, 13(2), 227-234.

Coelho, C. L. G., \& Correa, J. (2010). Desenvolvimento da compreensão leitora através do monitoramento da leitura. Psicologia: Reflexão e Crítica, 23(3), 575-581.

Espinosa, L. A., \& Tamayo, A. M. C. (2009). La enseñanza de estrategias metacognitivas para el mejoramiento de la comprensión lectora: estado de la cuestión. Pensamiento Psicológico, 5(12), 125-138.

Falk-Ross, F. C. (2002). Toward the new literacy: Changes in college students' reading comprehension strategies following reading/writing projects. Journal of Adolescent \& Adult Literacy, 45(4), 278-288.

Joly, M. C. R. A. (2004). Escala de estratégias metacognitivas de leitura - formato universitário (EMeL-U). Itatiba: Universidade São Francisco.

Joly, M. C. R. A. (2007). Escala de estratégias metacognitivas de leitura para universitários brasileiros: estudo de validade divergente. Universitas Psycologíca, 6(3), 507-521.

Joly, M. C. R. A., Cantalice, L. M., \& Vendramini, C. M. M. (2004). Evidências de validade de uma escala de estratégias de leitura para universitários. Interação em Psicologia, 8(2), 261-270.

Joly, M. C. R. A., \& Marini, J. A. S. (2006). Metacognição e Cloze na avaliação de dificuldades em leitura. In M. C. R. A. Joly \& C. Vectore (Orgs.). Questões de pesquisa e prática em psicologia escolar (pp. 13-36). São Paulo: Casa do Psicólogo.

Joly, M. C. R. A., Santos, L. M., \& Marini, J. A. S. (2006). Uso de estratégias de leitura por alunos do Ensino Médio. Paidéia, 16(34), 205-212.

Kopcke, H. F. (1997). Estratégias para desenvolver a metacognição e a compreensão de textos teóricos na universidade. Psicologia Escolar e Educacional, 1(2-3), 59-67.

Marini, J. A. S. (2006). Metacognição e leitura. Psicologia Escolar e Educacional, 10(2), 323-329.

Ministério da Educação. (2006). Programa de educação tutorial-PET: manual de orientações básicas. Brasília: Secretaria de Educação Superior.

Oliveira, K. L., \& Santos, A. A. A. (2005). Compreensão em leitura e avaliação da aprendizagem em universitários. Psicologia: Reflexão e Crítica, 18(1), 118-124.

Oliveira, K. L., \& Santos, A. A. A. (2008). Estudo de intervenção para a compreensão em leitura na universidade. Interação em Psicologia, 12(2), 169-177. 
Sampaio, I. S., \& Santos, A. A. A. (2002). Leitura e redação entre universitários: avaliação de um programa de intervenção. Psicologia em Estudo, 7(1), 31-38.

Santos, A. A. A., Suehiro, A. C. B., \& Oliveira, K. L. (2004). Habilidades em compreensão da leitura: um estudo com alunos de psicologia. Estudos de Psicologia, 21(2), 29-41.

Silva, E. M. T., \& Witter, G. P. (2008). Compreensão de texto e desempenho acadêmico em estudantes de psicologia. Estudos de Psicologia, 25(3), 395-403.

Song, M. (1998). Teaching reading strategies in ongoing EFL University Reading Classroom. Journal of English Language Teaching, 8, 41-54.

Witter, G. P. (1997). Leitura e universidade. In G. P. Witter (Org.). Psicologia: leitura e universidade (pp. 9-18). Campinas: Alínea.

Submissão: 9.1.2013

Aceitação: 10.9.2013 